\title{
MiR-138 Inhibits Tumor Growth Through Repression of EZH2 in Non-Small Cell Lung Cancer
}

\author{
Huijun Zhang ${ }^{\mathrm{a}, \mathrm{h}} \quad$ Hui Zhang ${ }^{\mathrm{b}, \mathrm{h}} \quad$ Mingchuan Zhao ${ }^{\mathrm{b}, \mathrm{h}} \quad$ Zhongwei Lv Xiaoping Zhang $^{\mathrm{c}}$ \\ Xiong Qin ${ }^{d}$ Heyong Wange Shaohua Wang ${ }^{f}$ Jinmei Su ${ }^{g}$ Xin Lva Hongcheng Liu ${ }^{b}$ \\ Weijia Du ${ }^{a}$ Wenyong Zhou ${ }^{b}$ Xiaofeng Chen ${ }^{b}$ Ke Fei ${ }^{b}$

\begin{abstract}
aDepartment of Anesthesiology, Shanghai Pulmonary Hospital, Tongji University, Shanghai; bDepartment of Thoracic Surgery, Shanghai Pulmonary Hospital, Tongji University, Shanghai; 'Department of Nuclear medicine, Tenth People's Hospital, Tongji University, Shanghai; 'Department of Thoracic Surgery, Tenth People's Hospital, Tongji University, Shanghai; ${ }^{e}$ Central Laboratory, Shanghai Pulmonary Hospital, Tongji University, Shanghai; ' Department of Thoracic Surgery, First People's Hospital, Shanghai Jiao Tong University, Shanghai; 9Department of Integrated Chinese Medicine and Western Medicine, Shanghai Pulmonary Hospital, Tongji University, Shanghai; hThese authors contributed equally to this work
\end{abstract}

\section{Key Words}

$\mathrm{miR}-138 \cdot \mathrm{EZH} 2 \cdot \mathrm{NSCLC}$

\begin{abstract}
Background/Aims: MicroRNAs (miRNAs) play important roles in tumorigenesis. We investigated the roles and mechanisms of miR-138 in human non-small cell lung cancer (NSCLC). Methods: The expression of miR-138 was first examined in NSCLC cell lines and tumour tissues by real-time PCR. The in vitro and in vivo functional effect of miR-138 was examined further. A luciferase reporter assay was conducted to confirm target association between miR-138 and the enhancer of zeste homolog 2 (EZH2). Results: miR-138 was frequently downregulated in NSCLC cells and tissues. Overexpression of miR-138 inhibited proliferation of NSCLC cells in vitro and tumor growth in vivo. The EZH2 oncogene, which is often overexpressed in various human cancers and acts as an important regulator of cell growth and tumor invasion, was identified as a novel target of miR-138. miR-138 can bind to the $3^{\prime}$ untranslated region ( $3^{\prime}$ UTR) of EZH2 and suppress the expression of EZH2 at both mRNA and protein levels. Furthermore, knockdown of EZH2 phenocopied the tumor suppressive effects of miR-138 in cell models, whereas ectopic expression of EZH2 rescued the suppressive effects of miR-138. Conclusion: These findings define a tumor suppressor function for miR-138 in NSCLC and further suggest that miR-138 may represent a potential therapeutic target for NSCLC patients.
\end{abstract}




\section{Cellular Physiology and Biochemistry}

Cell Physiol Biochem 2013;31:56-65

DOI: $10.1159 / 000343349$

Published online: January 15, 2013

C) 2013 S. Karger AG, Basel

www.karger.com/cpb

\section{Introduction}

Non-small cell lung cancer (NSCLC) including denocarcinomas, squamous cell carcinomas (SCC), and large cell carcinomas [1], is the most common cancer worldwide and accounts for approximately $80 \%$ of the total lung cancer cases [2]. Although recent advances in clinical diagnosis and therapeutic strategies, the overall prognosis for patients with NSCLC remains poor, with a 5-year overall survival rate of approximately 11\% [3]. Further understanding of the mechanisms of lung cancer development and progression is essential for improving the diagnosis, prevention and treatment of this disease.

MicroRNAs (miRNAs) are a class of endogenous small non-coding RNAs that repress gene expression by base pairing with messenger RNA (mRNA), leading to mRNA decay or translational repression [4-6]. Accumulating evidences have demonstrated that dysregulation of miRNAs may lead to alterations in diverse biological processes, such as proliferation, differentiation and apoptosis that are important in the development of cancer [7, 8]. Several deregulated miRNAs, inculding miR-126, miR-21, miR-200c, miR-145, miR-107, miR-185 and miR-101, have been shown to regulate NSCLC cell growth, apoptosis, migration and/or invasion [9-14]. These findings suggest that a dysfunction of miRNA may be associated with lung cancer. A recent study identified a series of miRNAs deregulated in SCC, including miR138 [15]. It has been shown that ectopic expression of miR-138 in hepatocellular carcinoma inhibits cell growth [16]. Liu and colleagues [17] reported that miR-138 could suppress invasion and promote apoptosis in head and neck squamous cell carcinoma cell lines. These data indicate a potential tumour suppressive function of miR-138. However, the role and the molecular mechanisms of miR-138 in NSCLC remain largely unknown.

In this study, we confirmed that miR-138 was frequently downregulated in NSCLC tissues and cell lines. Overexpression of miR-138 in NSCLC cells inhibited cell growth in vitro and tumor growth in nude mice. Furthermore, we showed that EZH2 was a direct functional target of miR-138 in NSCLC. Our findings provide a novel therapeutic strategy for treatment of NSCLC.

\section{Materials and Methods}

\section{Tissue samples and cell lines}

18 human NSCLC tissue samples and matched normal lung tissues were collected from Shanghai Pulmonary Hospital. Tissues were immediately snap-frozen in liquid nitrogen after surgery and stored at $-80^{\circ} \mathrm{C}$ until RNA extraction. Written informed consent was obtained from all patients. This study using human tissues was approved by Shanghai Pulmonal Hospital Ethical Committee. NSCLC cell lines A549, SPC-A1 (adenocarcinomas), SK-MES-1 (squamous carcinoma), H460 (large cell carcinoma) and normal human bronchial epithelial cell line (16HBE) were purchased from the Institute of Biochemistry and Cell Biology of the Chinese Academy of Sciences (Shanghai, China) and cultured in RPMI-1640 supplemented with $10 \%$ fetal bovine serum (FBS; Hyclone) with $100 \mathrm{IU} / \mathrm{ml}$ penicillin and $100 \mu \mathrm{g} / \mathrm{ml}$ streptomycin at $37^{\circ} \mathrm{C}$ with $5 \% \mathrm{CO}_{2}$.

\section{Total RNA isolation and quantitative real-time PCR ( $q R T-P C R)$}

Total RNA was extracted using TRIzol Reagent (Invitrogen, Carlsbad, CA, USA) according to the manufacturer's instructions. For analysis of mature miR-138 expression, total RNA was reversely transcribed using TaqMan MicroRNA Reverse Transcription Kit (Applied Biosystems, Foster City, CA, USA) along with miR-138-specific primers (Applied Biosystems). U6 snRNA was used as an endogenous control. For the detection of EZH2 mRNA, reverse transcription and qRT-PCR were performed using SYBR Green PCR master mix (Applied Biosystems) on an ABI 7500HT System. $\beta$-actin was amplified in parallel as an internal control. The primers used were as follows: EZH2, 5'-CAC TGA ACA GCA GCT TCC AGG-3' (Foward), 5'-AAG AAT GCA GGC TTT G CTCC-3' (Reverse); $\beta$-actin, $5^{\prime}$-CCG CAA ATG CTT CTA AAC CG-3' (Foward), $5^{\prime}$-AAA GCC ATG CCA ATC TCG TC-3' (Reverse). The relative expression level was computed using the $2^{-\Delta \Delta C t}$ analysis method. Experiments were performed in triplicate. 


\section{Cellular Physiology and Biochemistry}

Cell Physiol Biochem 2013;31:56-65

DOI: $10.1159 / 000343349$

Published online: January 15, 2013

(C) 2013 S. Karger AG, Basel

www.karger.com/cpb

Lentivirus production and infection

The pre-miR-138, EZH2 siRNA sequences or the coding sequences of EZH2 were cloned into pCDHCMV-MCS-EF1-coGFP vectors (System Biosciences, California, USA). The primers for pre-miR-138 were: 5'-CTC GAA TTC AGC AGC ACA AAG GCA TCT CT-3' (forward) and 5'-CCT GGA TCC GGG ATA AAC AGC AGC CTC AG-3' (reverse). The primers for the coding sequences of EZH2 were: $5^{\prime}$-CTA ACT AGT ATG GGC CAG ACT GGG AAG AAA T-3' (forward) and 5'-CGC GGA TCC TCA AGG GAT TTC CAT TTC TCT T-3' (reverse). EZH2 siRNA sequences were $5^{\prime}$-GAC TCT GAA TGC AGT TGC TTC AGT ACC C- $3^{\prime}$. Virus packaging and infection were performed according to standard protocols as recommended by the manufacturer. A549 and H460 cells $\left(1 \times 10^{5}\right)$ were infected with $1 \times 10^{7}$ lentivirus transducing units in the presence of $10 \mu \mathrm{g} / \mathrm{ml}$ polybrene (Sigma, St Louis, Missouri, USA).

Plasmid construction and luciferase reporter assay

To construct a luciferase reporter vector, the wild-type 3'UTR of EZH2 was amplified using genomic DNA from A549 and H460 cells as templates with the following primers: 5'-AGG TCT AGA CCT CTG AAA CAG CTG CCT TA-3' (forward) and 5'-CCT GAG CTC GCA TTA TTG CAA AAA TTC AC-3' (reverse). The corresponding mutant constructs were created by site-directed mutagenesis (QuikChange, Stratagene, La Jolla, CA). Both wild-type and mutant $3^{\prime}$ UTRs were cloned downstream of the luciferase gene in the pMIR-REPORT Luciferase vector. The constructs were verified by sequencing. For the luciferase assay, A549 and H460 cells were cultured in 24-well plates and cotransfected with $200 \mathrm{ng}$ of the luciferase reporter constructs and $20 \mathrm{ng}$ of the pRL-CMV renilla luciferase reporter construct in the presence of either miR-138 or miR-control. After $48 \mathrm{~h}$, luciferase activity was measured using the dual luciferase reporter assay system (Promega, Madison, WI, USA).

\section{Cell proliferation, apoptosis and cell cycle analysis}

Cell proliferation was monitored using Cell Proliferation Reagent Kit I (MTT) (Roche Applied Science). Briefly, cells (2000/well) overexpressing miR-138 or miR-control were seeded in 96-well plates. Cell proliferation was documented every $24 \mathrm{~h}$ following the manufacturer's protocol. The resulting absorbance at $570 \mathrm{~nm}$ was measured on a spectrophotometer. For analysis of apoptosis, cells were stained with FITCAnnexin V and Propidium iodide (PI), and then analyzed with a flow cytometry (FACScan $®$; BD Biosciences) equipped with a CellQuest software (BD Biosciences). For cell-cycle analysis, cells were suspended and stained with PI. Cell cycle assay was performed using an Epics Altra Flow Cytometer (BECKMAN Coulter, USA) and analyzed using EXP032 Multicomp and EXP032 v1.2 Analysis (BECKMAN Coulter, USA) software. All of the assays were conducted in triplicate.

\section{Western blotting}

Protein lysates were separated by $10 \%$ SDS-PAGE and then transferred to nitrocellulose membranes by electroblotting. The membranes were incubated with anti-EZH2 (1:500) or anti- $\beta$-actin (1:2000) antibodies (Cell Signaling Technology, Beverly, MA, USA) overnight at $4^{\circ} \mathrm{C}$ before subsequent incubation with secondary antibodies conjugated with horseradish peroxidase for $1 \mathrm{~h}$ at $37^{\circ} \mathrm{C}$. Protein was visualized using enhanced chemiluminescence reagent. The expression level of EZH2 protein was normalized to that of $\beta$-actin protein.

\section{Tumorigenicity assays in nude mice}

Male BALB/c nude mice aged 5 to 6 weeks were purchased from Shanghai Experimental Animal Center (Shanghai, China). Animal handling and experimental procedures were approved by the Animal Experiments Ethics Committee of Tongji University. For tumor growth assay, miR-138-overexpressed A549 cells $\left(1 \times 10^{6}\right.$ cells in $\left.100 \mu \mathrm{l}\right)$ were injected subcutaneously into the posterior flank of nude mice $(6$ mice per group). Tumor size was measured every 5 days. After 25 days, the mice were sacrificed and the tumors were weighed. Tumor volumes were calculated according to the following formula: Volume $=\left(A \times B^{2}\right) / 2$, where $A$ is the largest diameter and B is the shortest diameter.

\section{Statistical analysis}

Data are presented as mean \pm SD of at least 3 independent experiments. Differences between groups were evaluated by SPSS 13.0 statistical software with Student's $t$-test when comparing only two groups or 

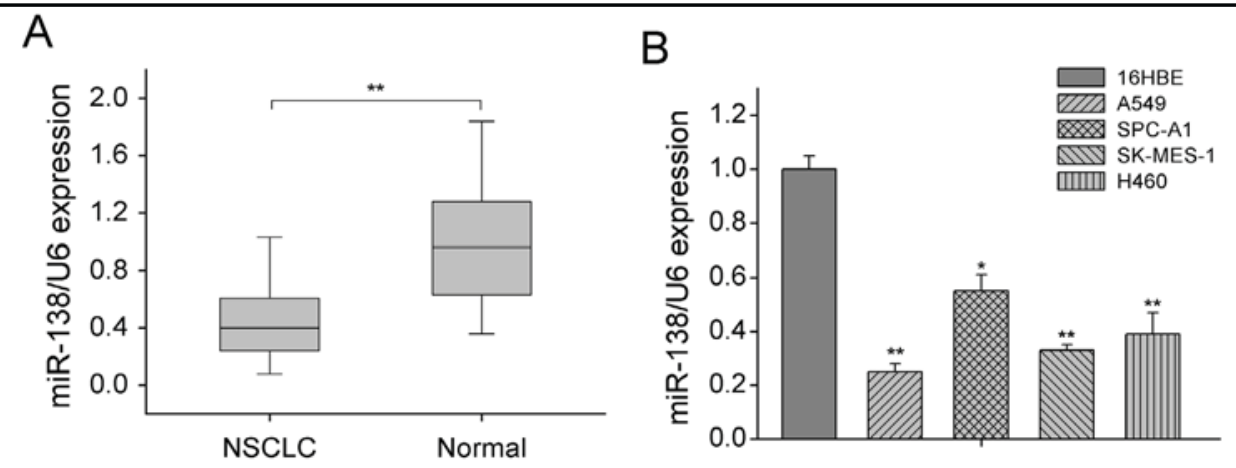

Fig. 1. MiR-138 is downregulated in NSCLC tissues and cell lines. (A) The expression of miR-138 was mesured by qRT-PCR in 18 paired NSCLC and adjacent normal tissues. Alteration of expression was shown as box plot presentations, with the y axis indicating miR-138 expression. The mean level of miR-138 expression in NSCLCs was significantly lower than that in normal tissues. (B) Expression levels of miR-138 in four NSCLC cell lines and 16HBE cells.

assessed by one-way analysis of variance when more than two groups were compared. For comparison of matched tissues, a paired Student's $t$-test was used to determine the statistical significance. Differences were considered statistically significant at $\mathrm{P}<0.05,{ }^{*} \mathrm{P}<0.05$ and ${ }^{* *} \mathrm{P}<0.01$.

\section{Results}

miR-138 is downregulated in NSCLC tissues and cell lines

To understand the biological role of miR-138 in NSCLC, qRT-PCR was first performed to measure miR-138 expression levels in 18 NSCLC tissue samples and 4 NSCLC cell lines. As shown in Fig. 1A, the expression levels of miR-138 were downregulated in NSCLC tissues as compared to matched normal tissues from the same patients. The expression of miR-138 in NSCLC cell lines was also significantly reduced as compared to normal human bronchial epithelial cell line (16HBE) (Fig. 1B). These results suggested that downregulation of miR138 might contribute to NSCLC carcinogenesis.

Overexpression of miR-138 suppresses proliferation, induces apoptosis and blocks G1/S transition in NSCLC cells

To investigate the effect of miR-138 on cell growth, we firstly constructed NSCLC cell lines with stable overexpression of miR-138 by infecting A549 and $\mathrm{H} 460$ cells with lentivirus particles. By using qRT-PCR, we verified significant increase of miR-138 expression in these established cell lines (Fig. 2A). These cells were then used to determine their growth curve, apoptosis and cell-cycle distribution. As shown in Fig. 2B, the growth curve showed a significant decrease of cell viability, examined by the MTT assay, in A549 and H460 cells as compared to negative control cells. Furthermore, miR-138 overexpression provoked a robust apoptotic response in NSCLC cells determined by FACS (Fig. 2C). Consistent with these observations, miR-138 overexpression enhanced the proportion of cells in G0/G1 phase, and decreased the number of cells in S phase in both A549 and H460 cells (Fig. 2D). Taken together, our results indicate that miR-138 suppressed cell growth by inducing apoptosis and G0/G1 arrest in NSCLC cells.

\section{EZH2 is a direct target of miR-138}

To explore the molecular mechanism of miR-138, we adopted bioinformatic algorithms (TargetScan and PicTar) to predict a large number of potential miR-138 target genes. Among them, EZH2 was found to have a putative miR-138 binding site within its $3^{\prime} U T R$, and this putative target site is highly conserved among the vertebrates. To verify whether 


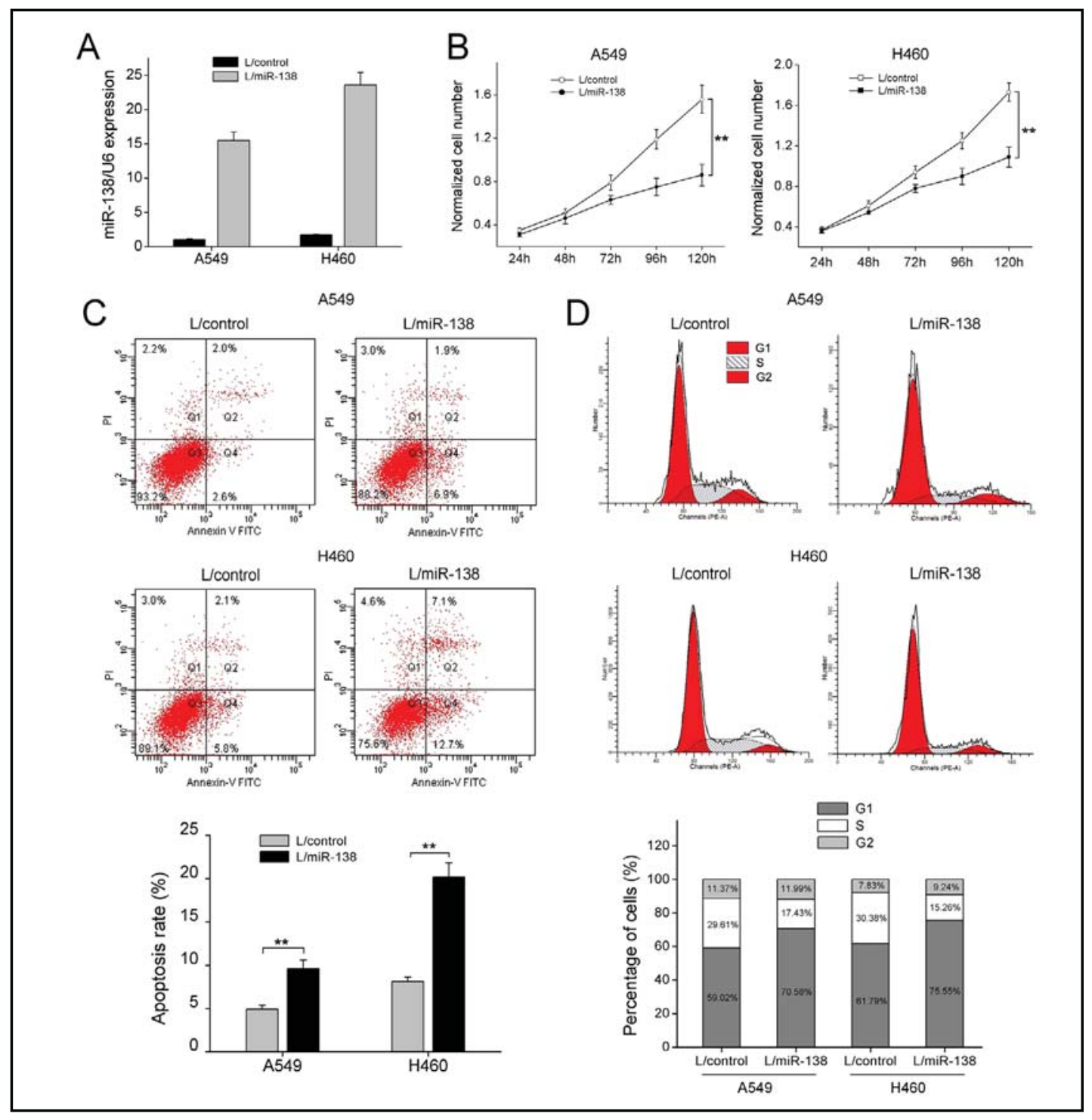

Fig. 2. MiR-138 overexpression suppresses cell proliferation, induces apoptosis and blocks G1/S transition in NSCLC cells. (A) qRT-PCR analysis of miR-138 in A549 and H460 cells which were infected with miR-138 lentivirus (indicated as L/miR-138) and control lentivirus (indicated as L/control). (B) Cell proliferation assay (MTT). (C) Cell apoptosis was evaluated by using Annexin V/Propidium Iodide-double staining. The apoptosis rates were the values of Q2+Q4 in each figure. (D) Representative histograms for cell-cycle distribution of A549 and H460 cells overexpressing miR-138.

EZH2 was a direct target of miR-138, the wild-type $3^{\prime}$ untranslated region (3'UTR) or the mutant sequence of EZH2 was fused directly downstream of the firefly luciferase gene (Fig. 3A). Then, miR-138-overexpressed A549 and H460 cells were transfected with wt or mut 3'UTR plasmids. As illustrated in Fig. 3B, miR-138 overexpression decreased the EZH2 $3^{\prime}$ UTR luciferase reporter activity, whereas no significant difference was detected when the 3 nucleotides in the miR-138 seed binding site of the EZH2 3'UTR were mutated. In addition, qRT-PCR and Western blot analyses showed that miR-138 overexpression significantly decreased the levels of EZH2 mRNA and protein expression in A549 and H460 cells (Fig. 3C, D). These results indicated that EZH2 was a direct target of miR-138 in NSCLC cells. 


\section{Cellular Physiology $\quad$ Cell Physiol Biochem 2013;31:56-65 and Biochemistry \begin{tabular}{l|l} 
DOI: 10.1159/000343349 & \\
Published online: January 15, 2013 & $\begin{array}{l}\text { 2 2013 S. Karger AG, Basel } \\
\text { www.karger.com/cpb }\end{array}$ \\
\hline
\end{tabular} Zhang/Zhang/Zhao et al.: MiR-138, a Tumor Suppressor in NSCLC}
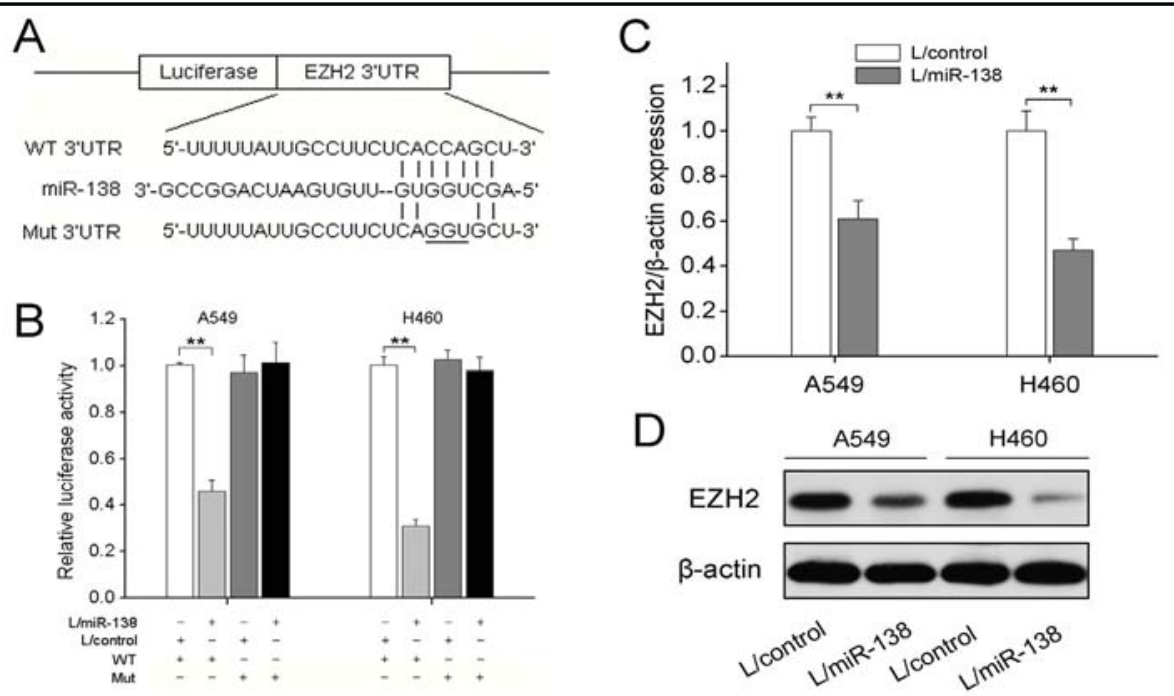

Fig. 3. MiR-138 downregulates EZH2 by interaction with its 3'UTR. (A) Diagram of EZH2 3'UTR-containing reporter construct. Mutations were generated at the three nucleotides in the EZH2 3'UTR. (B) The wild-type or mutant reporter plasmids were co-transfected into A549 and H460 cells which were infected by controllentivirus or miR-138-lentivirus. (C) The expression of EZH2 mRNA in A549 and H460 cells was examined by qRT-PCR. $\beta$-actin was used as an internal control. (D) The expression of EZH2 protein was examined by Western blotting. $\beta$-actin was used as an internal control.

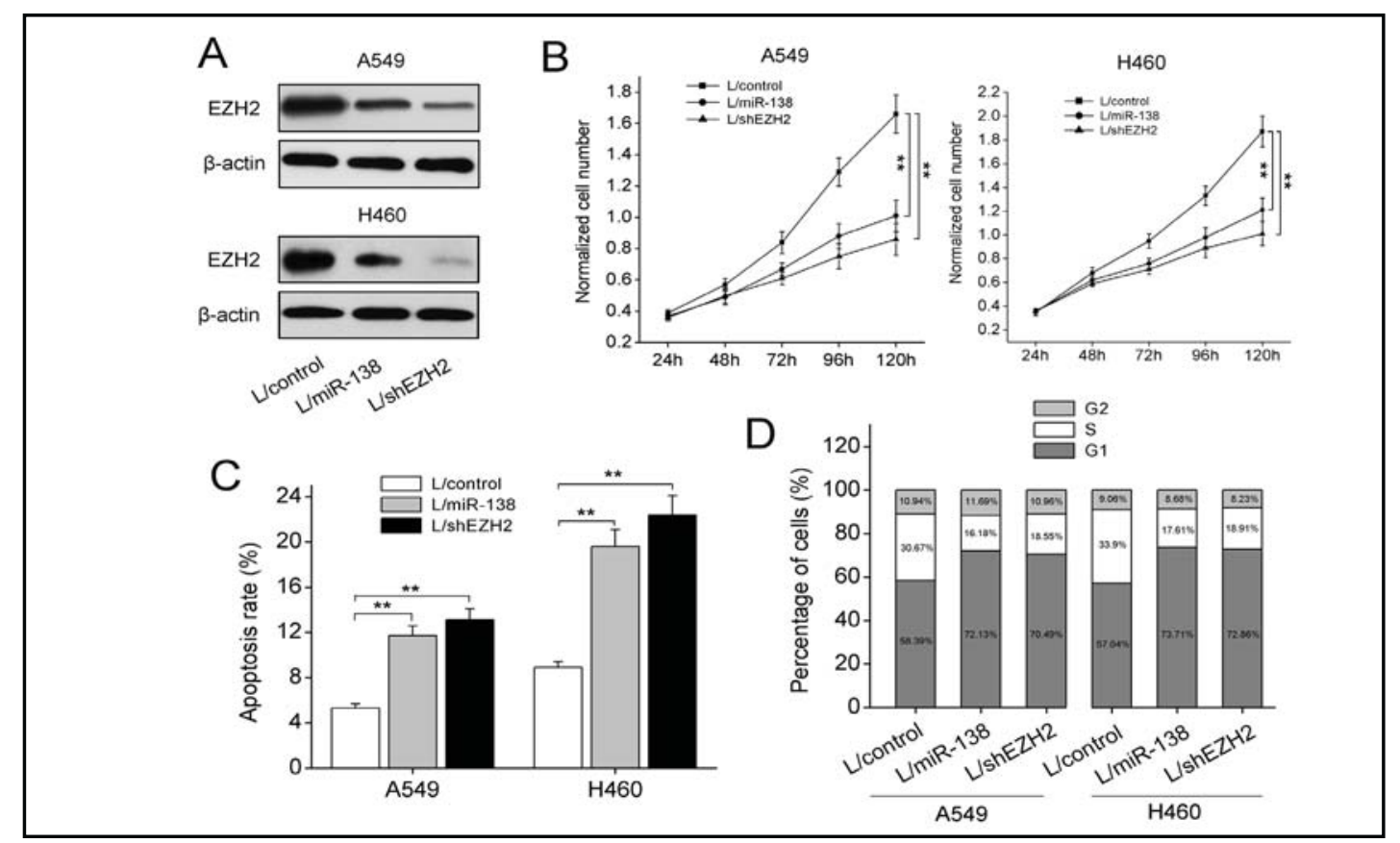

Fig. 4. Knockdown of EZH2 inhibites proliferation, induces apoptosis and suppresses cell-cycle progression similar to those induced by miR-138 in NSCLC cells. (A) EZH2 protein expression levels were determined by Western blotting. $\beta$-actin was used as an internal control. (B) Cell proliferation was assessed by the MTT assay. (C) Cell apoptosis was evaluated by using Annexin V/Propidium Iodide-double staining. (D) Cell-cycle distribution of A549 and H460 cells overexpressing miR-138 or downregulating EZH2.

\section{EZH2 mediates multiple biological actions of miR-138 in NSCLC cells}

To determine whether EZH2 acts as a critical mediator of miR-138's role in NSCLC cells, we performed targeted knockdown of EZH2 expression using RNA interference-based 


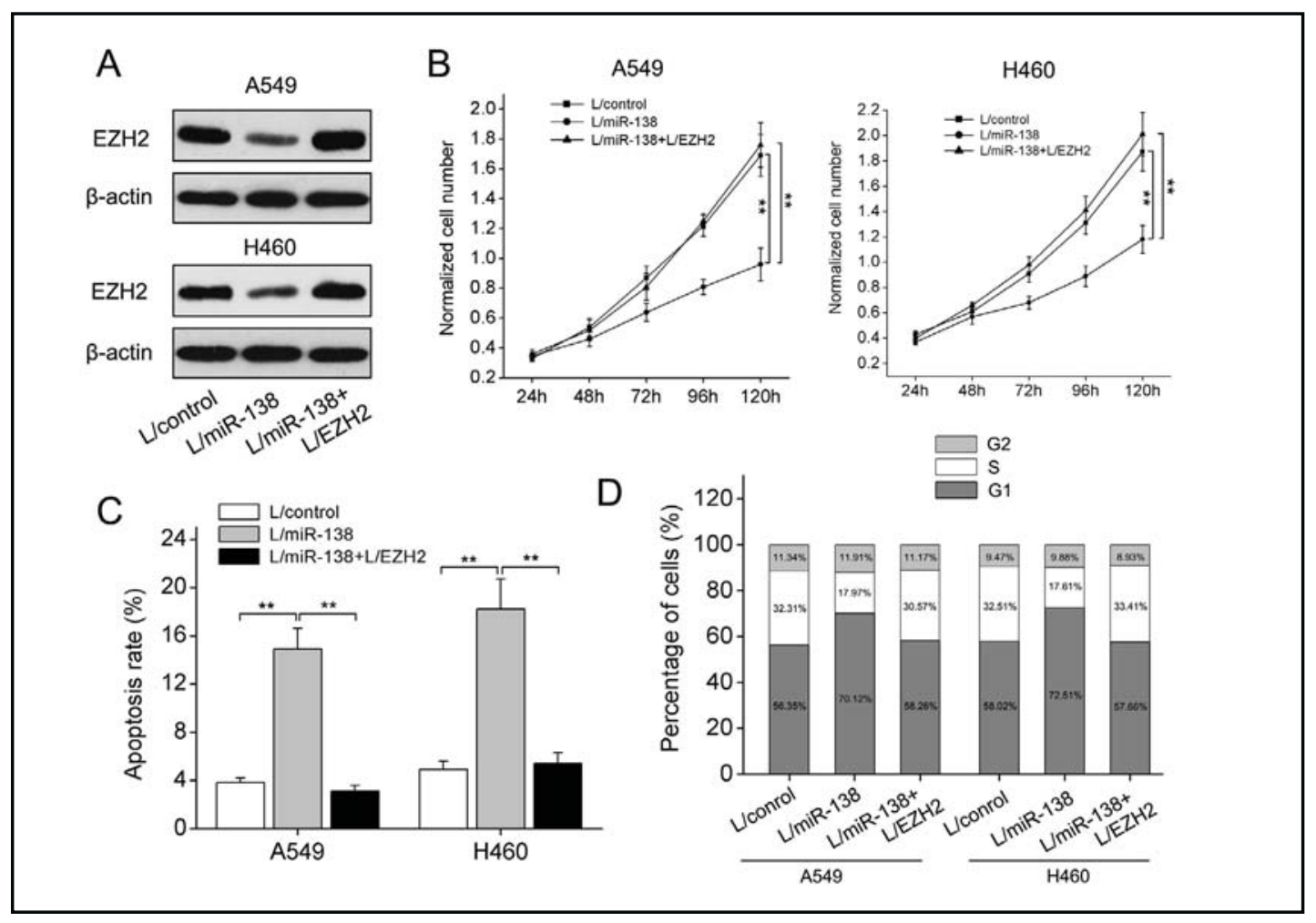

Fig. 5. Ectopic expression of EZH2 rescues miR-138-induced cell growth inhibition, apoptosis and cellcycle arrest. (A) EZH2 protein expression levels were determined by Western blotting. $\beta$-actin was used as an internal control. (B) Cell proliferation was assessed by the MTT assay. (C) Cell apoptosis was evaluated by using Annexin V/Propidium Iodide-double staining. (D) Cell-cycle distribution of A549 and H460 cells overexpressing miR-138 or coexpressing miR-138 and EZH2.

short hairpin RNA (shRNA) in A549 and H460 cells. As illustrated in Fig. 4A, Western blot analysis showed a significant reduction of EZH2 protein level in NSCLC cells. As expected, the reduction of EZH2 expression level led to a marked decrease in cell proliferation (Fig. 4B), accompanied by enhanced cell apoptosis (Fig. 4C) and G0/G1 cell-cycle arrest (Fig. 4D), similar to those induced by miR-138. Subsequently, we evaluated whether forced expression of EZH2 could rescue the suppressive effect of miR-138. miR-138-overexpressed A549 and H460 cells were infected with lentiviral constructs containing EZH2 gene (without the $3^{\prime}$ UTR region). These data showed that forced expression of EZH2 significantly rescued miR-138induced cell growth inhibition, apoptosis and cell-cycle arrest (Fig. 5). Taken together, these results indicate that miR-138 regulated NSCLC cell growth, at least in part, by downregulating EZH2.

miR-138 suppresses NSCLC tumor growth in nude mice

Next, we tested whether miR-138 could play an important role in vivo tumorigenesis. miR-138-overexpressed and control A549 cells were injected subcutaneously into the posterior flank of nude mice, respectively. After 25 days, significant decreases in tumor volume and weight were observed in miR-138-overexpressed tumors (Fig. 6A, B and C). Moreover, reduced EZH2 protein was confirmed in miR-138-overexpressed tumors by Western blotting (Fig. 6D). Taken together, these results demonstrated that stable overexpression of miR-138 inhibited the tumorigenicity of NSCLC cells in the nude mice xenograft model. 
A

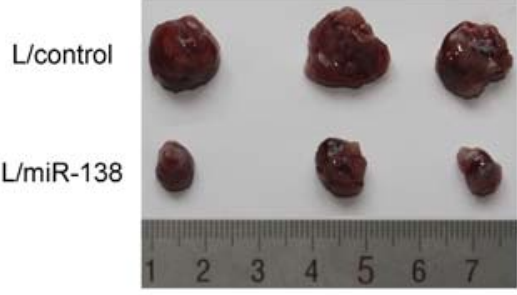

C

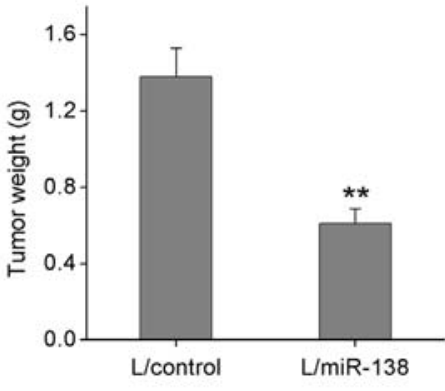

B

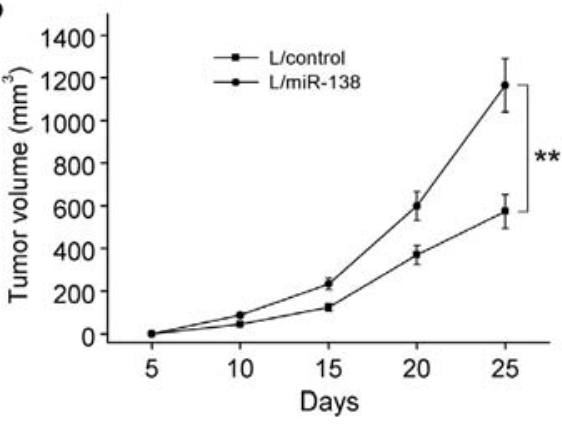

$\mathrm{D}$

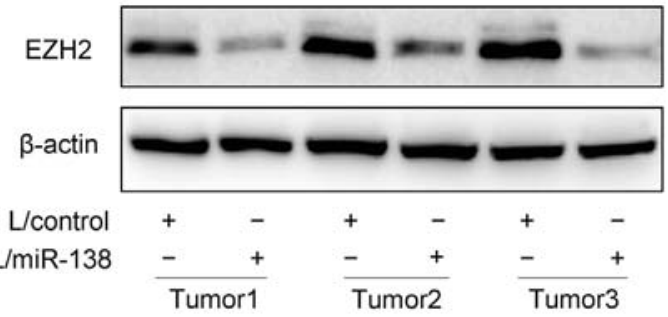

Fig. 6. miR-138 suppresses tumor growth of NSCLC cells in vivo. (A) Photography of xenograft tumor masses from nude mice. (B) The volume of xenograft tumors. (C) The weight of xenograft tumors. (D) Western blot analysis of EZH2 protein in miR-138-overexpressed and control xenograft tumor tissues.

\section{Discussion}

Although deregulation of miRNAs in many types of human cancers have been identified, the molecular mechanisms by which miRNAs regulate the processes of diverse cancers are still unclear $[18,19]$. Recently, Tan et al showed that the expression of miR-138 was decreased in SCC by microRNA microarray analysis [15], but no data are available about its functions in NSCLC. In the present study, we investigated the potential suppressive roles and the mechanisms of miR-138 in the development and progression of NSCLC through cell models and tissue samples.

Previous studies have indicated that miR-138 plays critical roles in tumorigenesis. For instance, a decreased aberrant miR-138 expression is associated with enhanced cell proliferation and invasion in OSCC [17, 20]. Ectopic transfection of miR-138 in TSCC cells reduced the expression of GNAI2, which led to reduced proliferation, cell cycle arrest and apoptosis [21]. It was also observed that miR-138 may function as a nasopharyngeal carcinoma (NPC) suppressor by inhibiting CCND1 expression [22]. However, the functions of miR-138 in NSCLC is little known. To reveal the role of miR-138 in NSCLC, we tested the effect of miR-138 on cell growth. Our results indicated that overexpression of miR-138 significantly affected cell biological behaviors, that is, suppressing cell proliferation, enhancing apoptosis and inducing G0/G1 cell-cycle arrest. Furthermore, we found that stable overexpression of miR-138 could inhibit the tumorigenicity in a xenograft model. Taken together, these findings suggest that miR-138 functions as a tumor suppressor in NSCLC.

To explore the molecular mechanisms of miR-138-induced growth inhibition on NSCLC progression, we used bioinformatic algorithms to predict gene targets for miR-138. Although many cancer-associated genes were predicted, Enhancer of zeste homolog 2 (EZH2) was selected as a candidate target of miR-138 for further research because of its promoting role in the initiation and propagation of a number of cancers, including prostate, bladder, gastric, 


\section{Cellular Physiology and Biochemistry}

Cell Physiol Biochem 2013;31:56-65

DOI: $10.1159 / 000343349$

Published online: January 15, 2013

Zhang/Zhang/Zhao et al.: MiR-138, a Tumor Suppressor in NSCLC

hepatocellular, and nasopharyngeal cancers [23-27]. A recent study has identified EZH2 as a target of miR-138 in thermoregulatory system development and functioning in chicks [28]. In our studies, we confirmed that EZH2 is a direct target of miR-138 in NSCLC cells, and this conclusion is supported by the following observations: miR-138-binding site is identified in the 3'UTR of EZH2 mRNA; miR-138 overexpression decreased EZH2 3'UTR luciferase report activity and this effect was abolished by mutation of the miR-138 seed binding site; miR138 overexpression reduced the expression of EZH2 mRNA and protein. To further reveal the roles of EZH2 in NSCLC, we silenced EZH2 by specific RNAi in NSCLC cells, and found that EZH2 knockdown phenocopied the tumor suppressive effects of miR-138 in cell models, whereas ectopic expression of EZH2 significantly rescued the suppressive effects of miR138. Together, these results suggest that miR-138 modulates NSCLC cell growth, at least in part, by directly targeting EZH2.

In summary, our results identify that miR-138 as a potential tumor suppressor could inhibit cell proliferation, induce cell apoptosis and G0/G1 cell-cycle arrest, by targeting EZH2 in NSCLC cells. Our data suggest that miR-138 could be employed as a potential therapeutic target for miRNA-based NSCLC therapy.

\section{Acknowledgements}

This work was supported by Grants from National Science Foundation of China (No. 30872553 ) and Shanghai Science and Technology Committee (No. 10JC1419200).

\section{References}

1 Liu XH, Lu KH, Wang KM, Sun M, Yang JS, Zhang EB, Yin DD, Liu ZL, Zhou J, Liu ZJ, De W, Wang ZX: MicroRNA-196a promotes non-small cell lung cancer cell proliferation and invasion through targeting HOXA5. BMC Cancer 2012;12:348-349.

- Jemal A, Siegel R, Xu J, Ward E: Cancer statistics. CA Cancer J Clin 2010;60:277-300.

-3 Verdecchia A, Francisci S, Brenner H, Gatta G, Micheli A, Mangone L, Kunkler I: Recent cancer survival in Europe: a 2000-02 period analysis of EUROCARE-4 data. Lancet Oncol 2007;8:784-796.

4 Ambros V: The functions of animal microRNAs. Nature 2004;431:350-355.

5 Bartel DP: MicroRNAs: genomics, biogenesis, mechanism, and function. Cell 2004;116:281-297.

6 He L, Hannon GJ: MicroRNAs: small RNAs with a big role in gene regulation. Nat Rev Genet 2004;5:522531.

7 Hwang HW, Mendell JT: MicroRNAs in cell proliferation, cell death, and tumorigenesis. Br J Cancer 2007;96:R40-R44.

8 Maatouk D, Harfe B: MicroRNAs in development. Sci World J 2006;6:1828-1840.

-9 Crawford M, Brawner E, Batte K, Yu L, Hunter MG, Otterson GA, Nuovo G, Marsh CB, Nana-Sinkam SP: MicroRNA-126 inhibits invasion in non-small cell lung carcinoma cell lines. Biochem Biophys Res Commun 2008:373:607-612.

10 Zhang JG, Wang JJ, Zhao F, Liu Q Jiang K, Yang GH: MicroRNA-21 (miR-21) represses tumor suppressor PTEN and promotes growth and invasion in non-small cell lung cancer (NSCLC). Clin Chim Acta 2010;411:846-852.

-11 Ceppi P, Mudduluru G, Kumarswamy R, Rapa I, Scagliotti GV, Papotti M, Allgayer H: Loss of miR-200c expression induces an aggressive, invasive, and chemoresistant phenotype in non-small cell lung cancer. Mol Cancer Res 2010;8:1207-1216.

12 Chen Z, Zeng H, Guo Y, Liu P, Pan H, Deng A, Hu J: miRNA-145 inhibits non-small cell lung cancer cell proliferation by targeting c-Myc. J Exp Clin Cancer Res 2010;29:151-155. 


\section{Cellular Physiology and Biochemistry}

Cell Physiol Biochem 2013;31:56-65

Zhang/Zhang/Zhao et al.: MiR-138, a Tumor Suppressor in NSCLC

13 Takahashi Y, Forrest AR, Maeno E, Hashimoto T, Daub CO, Yasuda J: MiR-107 and MiR-185 can induce cell cycle arrest in human non small cell lung cancer cell lines. PLoS One 2009;4:e6677.

14 Zhang JG, Guo JF, Liu DL, Liu Q, Wang JJ: MicroRNA-101 Exerts Tumor-Suppressive Functions in Non-small Cell Lung Cancer through Directly Targeting Enhancer of Zeste Homolog 2. J Thorac Oncol 2011;6:671-678.

15 Tan X, Qin W, Zhang L, Hang J, Li B, Zhang C, Wan J, Zhou F, Shao K, Sun Y, Wu J, Zhang X, Qiu B, Li N, Shi S, Feng X, Zhao S, Wang Z, Zhao X, Chen Z, Mitchelson K, Cheng J, Guo Y, He J: A 5-MicroRNA Signature for Lung Squamous Cell Carcinoma Diagnosis and hsa-miR-31 for Prognosis. Clin Cancer Res 2011;17:68026811.

16 Wang W, Zhao LJ, Tan YX, Ren H, Qi ZT: MiR-138 induces cell cycle arrest by targeting cyclin D3 in hepatocellular carcinoma. Carcinogenesis 2012;33:1113-1120.

17 Liu X, Jiang L, Wang A, Yu J, Shi F, Zhou X: MicroRNA-138 suppresses invasion and promotes apoptosis in head and neck squamous cell carcinoma cell lines. Cancer Lett 2009;286:217-222.

-18 Lu J, Getz G, Miska EA, Alvarez-Saavedra E, Lamb J, Peck D, Sweet-Cordero A, Ebert BL, Mak RH, Ferrando AA, Downing JR, Jacks T, Horvitz HR, Golub TR: MicroRNA expression profiles classify human cancers. Nature 2005;435:834-838.

19 Volinia S, Calin GA, Liu CG, Ambs S, Cimmino A, Petrocca F, Visone R, Iorio M, Roldo C, Ferracin M, Prueitt RL, Yanaihara N, Lanza G, Scarpa A, Vecchione A, Negrini M, Harris CC, Croce CM: A microRNA expression signature of human solid tumors defines cancer gene targets. Proc Natl Acad Sci USA 2006;103:2257-2261.

-20 Liu X, Chen Z, Yu J, Xia J, Zhou X: MicroRNA profiling and head and neck cancer. Comp Funct Genomics 2009;2009:837514.

-21 Jiang L, Dai Y, Liu X, Wang C, Wang A, Chen Z, Heidbreder CE, Kolokythas A, Zhou X: Identification and experimental validation of $G$ protein alpha inhibiting activity polypeptide 2 (GNAI2) as a microRNA-138 target in tongue squamous cell carcinoma. Hum Genet 2011;129:189-197.

-22 Liu X, Lv XB, Wang XP, Sang Y, Xu S, Hu K, Wu M, Liang Y, Liu P, Tang J, Lu WH, Feng QS, Chen LZ, Qian CN, Bei JX, Kang T, Zeng YX: MiR-138 suppressed nasopharyngeal carcinoma growth and tumorigenesis by targeting the CCND1 oncogene. Cell Cycle 2012;11:2495-2506.

-23 Chen Y, Lin MC, Wang H, Chan CY, Jiang L, Ngai SM, Yu J, He ML, Shaw PC, Yew DT, Sung JJ, Kung HF: Proteomic analysis of EZH2 downs tream target proteins in hepatocellular carcinoma. Proteomics 2007;7:3097-3104.

-24 Lu J, He ML, Wang L, Chen Y, Liu X, Dong Q, Chen YC, Peng Y, Yao KT, Kung HF, Li XP: MiR-26a Inhibits Cell Growth and Tumorigenesis of Nasopharyngeal Carcinoma through Repression of EZH2. Cancer Res 2011;71:225-233.

25 Bryant RJ, Cross NA, Eaton CL, Hamdy FC, Cunliffe VT: EZH2 promotes proliferation and invasiveness of prostate cancer cells. Prostate 2007;67:547-556.

26 Zhang YB, Niu HT, Chang JW, Dong GL, Ma XB: EZH2 silencing by RNA interference inhibits proliferation in bladder cancer cell lines. Eur J Cancer Care (Engl) 2011:20:106-112.

27 Matsukawa Y, Semba S, Kato H, Ito A, Yanagihara K, Yokozaki H: Expression of the enhancer of zeste homolog 2 is correlated with poor prognosis in human gastric cancer. Cancer Sci 2006;97:484-491.

-28 Kisliouk T, Yosefi S, Meiri N: MiR-138 inhibits EZH2 methyltransferase expression and methylation of histone H3 at lysine 27, and affects thermotolerance acquisition. Eur J Neurosci 2011;33:224-235. 